Supplement of Hydrol. Earth Syst. Sci., 24, 1211-1225, 2020

https://doi.org/10.5194/hess-24-1211-2020-supplement

(c) Author(s) 2020. This work is distributed under

the Creative Commons Attribution 4.0 License.

(c) (1)

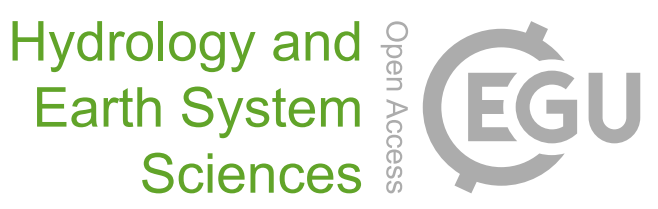

Supplement of

\title{
Responses of soil water storage and crop water use efficiency to changing climatic conditions: a lysimeter-based space-for-time approach
}

Jannis Groh et al.

Correspondence to: Jannis Groh (j.groh@fz-juelich.de, groh@zalf.de)

The copyright of individual parts of the supplement might differ from the CC BY 4.0 License. 
Table S1: Basic soil texture information for soils from Bad Lauchstädt (BL), Sauerbach (Sb), Dedelow (Dd), and Selhausen (Se). Please note the soil monoliths from Dedelow have a varying soil depth to the clay illuviation horizon (Bt) and to the marly, illitic glacial till (Chorizon). The profile for Dedelow describes the soil profile from soil monolith Dd_1.

\begin{tabular}{|c|c|c|c|c|c|c|}
\hline Soil & Horizon & Upper depth & Lower depth & Sand & Silt & Clay \\
\hline & & $\mathrm{m}$ & $\mathrm{m}$ & $\%$ & $\%$ & $\%$ \\
\hline \multirow[t]{6}{*}{$\mathrm{BL}$} & Ap & 0 & 0.23 & 5 & 73 & 22 \\
\hline & Axh1 & 0.23 & 0.33 & 4 & 83 & 13 \\
\hline & Axh2 & 0.33 & 0.70 & 4 & 82 & 14 \\
\hline & $\mathrm{C} 1$ & 0.70 & 1.10 & 6 & 67 & 27 \\
\hline & $\mathrm{C} 2$ & 1.10 & 1.45 & 5 & 72 & 23 \\
\hline & C3 & 1.45 & $>1.50$ & 65 & 16 & 19 \\
\hline \multirow[t]{4}{*}{$\mathrm{Sb}$} & Ap & 0 & 0.26 & 13 & 62 & 25 \\
\hline & M1 & 0.26 & 0.75 & 15 & 70 & 15 \\
\hline & M2 & 0.75 & 1.04 & 11 & 74 & 15 \\
\hline & M3 & 1.04 & 1.5 & 6 & 75 & 19 \\
\hline \multirow[t]{4}{*}{$\mathrm{Dd}$} & Ap & 0.00 & 0.30 & 54 & 31 & 15 \\
\hline & $\mathrm{Al}+\mathrm{Bt}$ & 0.30 & 0.42 & 51 & 34 & 15 \\
\hline & $\mathrm{Bt}$ & 0.42 & 0.90 & 52 & 23 & 25 \\
\hline & elCcv & 0.80 & 1.50 & 59 & 29 & 12 \\
\hline \multirow[t]{3}{*}{$\mathrm{Se}$} & Ap & 0 & 0.23 & 15 & 68 & 17 \\
\hline & $\mathrm{Al}-\mathrm{Bv}$ & 0.23 & 0.68 & 15 & 63 & 22 \\
\hline & II-Btv & 0.68 & $>1.50$ & 17 & 61 & 22 \\
\hline
\end{tabular}


Table S2: Average observed soil water flux at $1.5 \mathrm{~m}$ soil depth of soils from Bad Lauchstädt (BL), Sauerbach (Sb), Dedelow (Dd), and Selhausen (Se) under a dry climate Bad Lauchstädt (BL) and wet climate Selhausen (Se).

\begin{tabular}{|l|l|l|l|l|l|l|l|l|l|}
\hline \multirow{2}{*}{ Origin } & Transfer & $2011^{*}$ & 2012 & 2013 & 2014 & 2015 & 2016 & 2017 & Average $^{\#}$ \\
\cline { 3 - 10 } & & $\mathrm{mm}$ & $\mathrm{mm}$ & $\mathrm{mm}$ & $\mathrm{mm}$ & $\mathrm{mm}$ & $\mathrm{mm}$ & $\mathrm{mm}$ & $\mathrm{mm}$ \\
\hline $\mathrm{BL}-$ & -8 & -91 & -91 & 119 & -16 & -35 & -53 & -28 \\
\hline $\mathrm{Sb}$ & $\mathrm{BL}$ & 17 & -18 & -97 & 93 & -38 & -32 & -12 & -17 \\
\hline $\mathrm{Dd}$ & $\mathrm{BL}$ & 33 & -78 & -63 & 164 & -34 & -52 & -44 & -18 \\
\hline $\mathrm{Se}$ & $\mathrm{BL}$ & 17 & -19 & -99 & 96 & -27 & -41 & -19 & -18 \\
\hline $\mathrm{BL}$ & $\mathrm{Se}$ & 31 & 61 & -57 & 206 & 7 & 94 & -14 & 50 \\
\hline $\mathrm{Sb}$ & $\mathrm{Se}$ & 19 & 28 & -28 & 132 & -8 & 177 & 46 & 58 \\
\hline $\mathrm{Dd}$ & $\mathrm{Se}$ & 48 & 66 & 76 & 284 & 41 & 223 & 27 & 119 \\
\hline $\mathrm{Se}-$ & 28 & 30 & -5 & 142 & 20 & 68 & 40 & 49 \\
\hline$*$ April-December; $2012-2017$ & & & & & \\
\hline
\end{tabular}

15 
Table S3: Site management information on seasonal crop type, sowing and harvesting date, crop growth length N-fertilizer and number of fungicide applications at Bad Lauchstädt and Selhausen. Calcium ammonium nitrate (KAS) was mainly us as $N$-fertilizer. Except for 2013 in Selhausen, where ammonium sulphate nitrate (ASS) instead of KAS was used.

\begin{tabular}{|c|c|c|c|c|c|c|c|c|c|c|}
\hline \multirow[t]{2}{*}{ Crop } & \multicolumn{5}{|c|}{ Bad Lauchstädt } & \multicolumn{5}{|l|}{ Selhausen } \\
\hline & $\begin{array}{l}\text { Sowing } \\
\text { YY/mm } \\
\text { /dd }\end{array}$ & $\begin{array}{l}\text { Harvest } \\
\text { YY/mm } \\
\text { /dd }\end{array}$ & $\begin{array}{l}\text { Duration } \\
\text { [days] }\end{array}$ & $\begin{array}{l}\text { N-Fertilizer } \\
(\text { KAS) } \\
\mathrm{kg} \mathrm{N} \mathrm{ha}^{-1}\end{array}$ & $\begin{array}{l}\text { Number of } \\
\text { fungicide } \\
\text { applications }\end{array}$ & $\begin{array}{l}\text { Sowing } \\
\text { YY/mm/ } \\
\text { dd }\end{array}$ & $\begin{array}{l}\text { Harvest } \\
\mathrm{YY} / \mathrm{mm} / \mathrm{dd}\end{array}$ & $\begin{array}{l}\text { Duration } \\
\text { [days] }\end{array}$ & $\begin{array}{l}\text { N-Fertilizer } \\
\text { (KAS) } \\
\mathrm{kg} \mathrm{N} \mathrm{ha}^{-1}\end{array}$ & $\begin{array}{l}\text { Number of } \\
\text { fungicide } \\
\text { applications }\end{array}$ \\
\hline Pea & $\begin{array}{l}11 / \\
05 / 04\end{array}$ & $\begin{array}{l}11 / \\
08 / 11\end{array}$ & 99 & & 0 & $11 / 06 / 01$ & $11 / 08 / 25$ & 85 & & 0 \\
\hline $\begin{array}{l}\text { Winter } \\
\text { Barley }\end{array}$ & $\begin{array}{l}11 / \\
09 / 30\end{array}$ & $\begin{array}{l}12 / \\
07 / 12\end{array}$ & 278 & 145 & 3 & $11 / 10 / 14$ & $12 / 07 / 10$ & 270 & 50 & $\S$ \\
\hline $\begin{array}{l}\text { Winter } \\
\text { Canola }\end{array}$ & $\begin{array}{l}12 / \\
08 / 27\end{array}$ & $\begin{array}{l}13 / \\
07 / 23\end{array}$ & 330 & 210 & 2 & $12 / 09 / 18$ & $13 / 07 / 25$ & 310 & $130^{\#}$ & 1 \\
\hline Oat & $\begin{array}{l}14 / \\
03 / 13\end{array}$ & $\begin{array}{l}14 / \\
06 / 03\end{array}$ & 82 & 60 & 0 & $14 / 03 / 05$ & $14 / 06 / 03$ & 90 & 60 & 0 \\
\hline $\begin{array}{l}\text { Winter } \\
\text { Wheat }\end{array}$ & $\begin{array}{l}14 / \\
10 / 13\end{array}$ & $\begin{array}{l}15 / \\
07 / 28\end{array}$ & 288 & 60 & 2 & $14 / 10 / 07$ & $15 / 07 / 21$ & 279 & 90 & 2 \\
\hline $\begin{array}{l}\text { Winter } \\
\text { Barley }\end{array}$ & $\begin{array}{ll}15 / & 09 / \\
22 & \end{array}$ & $\begin{array}{l}16 / \\
06 / 30\end{array}$ & 282 & 100 & 3 & $15 / 10 / 07$ & $16 / 07 / 08$ & 275 & 80 & 2 \\
\hline $\begin{array}{l}\text { Winter } \\
\text { Rye }\end{array}$ & $\begin{array}{l}16 / \\
10 / 05\end{array}$ & $\begin{array}{l}17 / \\
07 / 17\end{array}$ & 285 & 100 & 2 & $16 / 10 / 11$ & $17 / 07 / 21$ & 283 & 78 & 0 \\
\hline
\end{tabular}

\# ASS; § no data available 
20 Table S4: Average and coefficient of variation (CV) of yield for soil from Bad Lauchstädt (BL), Dedelow (Dd), Sauerbach (Sb), Selhausen (Se) under dry (BL) and wet climate (Se). Origin describes the location, where the soil was taken from and the location where the soil was transferred to, where the soil was transferred to. The value in the brackets describes the variability of yield for each soil type (standard deviation from three replicates).

\begin{tabular}{|c|c|c|c|c|c|c|c|c|c|c|c|c|c|c|}
\hline \multicolumn{2}{|c|}{ Test site } & \multicolumn{2}{|c|}{2011} & \multicolumn{2}{|c|}{2012} & \multicolumn{2}{|c|}{2013} & \multirow{2}{*}{$\begin{array}{r}2014 \\
\text { Oat }\end{array}$} & \multicolumn{2}{|l|}{2015} & \multicolumn{2}{|l|}{2016} & \multicolumn{2}{|l|}{2017} \\
\hline Origin & Transfer & \multicolumn{2}{|c|}{ Pea } & \multicolumn{2}{|c|}{ Winter barley } & \multicolumn{2}{|c|}{ Winter canola } & & \multicolumn{2}{|c|}{ Winter wheat } & \multicolumn{2}{|c|}{ Winter barley } & \multicolumn{2}{|c|}{ Winter rye } \\
\hline & & $\mathrm{t} \mathrm{ha}^{-1}$ & $\mathrm{CV} \%$ & $\mathrm{tha}^{-1}$ & $\mathrm{CV} \%$ & $\mathrm{t} \mathrm{ha}^{-1}$ & $\mathrm{CV} \%$ & $\mathrm{t} \mathrm{ha}^{-1}$ & $\mathrm{t} \mathrm{ha}^{-1}$ & $\mathrm{CV} \%$ & $\mathrm{tha}^{-1}$ & $\mathrm{CV} \%$ & $\mathrm{tha}^{-1}$ & CV \% \\
\hline $\mathrm{BL}$ & & $\begin{array}{l}2.20 \\
( \pm 0.44)\end{array}$ & 20 & $\begin{array}{l}9.30 \\
( \pm 1.01)\end{array}$ & 11 & $\begin{array}{l}7.01 \\
( \pm 1.60)\end{array}$ & 23 & $\#$ & $\begin{array}{l}7.81 \\
( \pm 0.42)\end{array}$ & 5 & $\begin{array}{l}8.46 \\
( \pm 0.23)\end{array}$ & 3 & $\begin{array}{l}7.35 \\
( \pm 1.35)\end{array}$ & 18 \\
\hline $\mathrm{Dd}$ & $\mathrm{BL}$ & $\begin{array}{l}1.11 \\
( \pm 0.48)\end{array}$ & 43 & $\begin{array}{l}8.79 \\
( \pm 0.71)\end{array}$ & 8 & $\begin{array}{l}5.64 \\
( \pm 0.50)\end{array}$ & 9 & $\#$ & $\begin{array}{l}5.90 \\
( \pm 0.26)\end{array}$ & 4 & $\begin{array}{l}9.42 \\
( \pm 0.81)\end{array}$ & 9 & $\begin{array}{l}7.74 \\
( \pm 0.97)\end{array}$ & 13 \\
\hline $\mathrm{Sb}$ & $\mathrm{BL}$ & $\begin{array}{l}2.44 \\
( \pm 0.92)\end{array}$ & 38 & $\begin{array}{l}10.28 \\
( \pm 2.47)\end{array}$ & 24 & $\begin{array}{l}4.65 \\
( \pm 0.61)\end{array}$ & 13 & $\#$ & $\begin{array}{l}8.33 \\
( \pm 0.37)\end{array}$ & 4 & $\begin{array}{l}11.36 \\
( \pm 0.46)\end{array}$ & 4 & $\begin{array}{l}6.83 \\
( \pm 0.92)\end{array}$ & 13 \\
\hline $\mathrm{Se}$ & $\mathrm{BL}$ & $\begin{array}{l}0.99 \\
( \pm 0.17)\end{array}$ & 17 & $\begin{array}{l}7.76 \\
( \pm 1.50)\end{array}$ & 19 & $\begin{array}{l}5.07 \\
( \pm 0.82)\end{array}$ & 16 & $\#$ & $\begin{array}{l}6.36 \\
( \pm 0.36)\end{array}$ & 6 & $\begin{array}{l}8.42 \\
( \pm 0.66)\end{array}$ & 8 & $\begin{array}{l}5.45 \\
( \pm 0.53)\end{array}$ & 10 \\
\hline $\mathrm{BL}$ & $\mathrm{Se}$ & $\begin{array}{l}1.87 \\
( \pm 0.45)\end{array}$ & 24 & $\begin{array}{l}8.43 \\
( \pm 1.29)\end{array}$ & 15 & $\begin{array}{l}1.32 \\
( \pm 0.80)\end{array}$ & 62 & $\#$ & $\begin{array}{l}5.66 \\
( \pm 0.88)\end{array}$ & 16 & $\begin{array}{l}5.14 \\
( \pm 0.05)\end{array}$ & 1 & $\begin{array}{l}4.77 \\
( \pm 0.44)\end{array}$ & 9 \\
\hline $\mathrm{Dd}$ & $\mathrm{Se}$ & $\begin{array}{l}1.13 \\
( \pm 0.64)\end{array}$ & 57 & $\begin{array}{l}4.02 \\
( \pm 2.31)\end{array}$ & 57 & $\begin{array}{l}2.21 \\
( \pm 0.38)\end{array}$ & 17 & \# & $\begin{array}{l}4.73 \\
( \pm 0.83)\end{array}$ & 18 & $\begin{array}{l}3.51 \\
( \pm 2.31)\end{array}$ & 1 & $\begin{array}{l}4.77 \\
( \pm 0.44)\end{array}$ & 9 \\
\hline $\mathrm{Sb}$ & $\mathrm{Se}$ & $\begin{array}{l}1.90 \\
( \pm 0.22)\end{array}$ & 12 & $\begin{array}{l}5.06 \\
( \pm 3.56)\end{array}$ & 70 & $\begin{array}{l}3.66 \\
( \pm 1.03)\end{array}$ & 28 & $\#$ & $\begin{array}{l}5.67 \\
( \pm 0.14)\end{array}$ & 2 & $\begin{array}{l}5.01 \\
( \pm 2.02)\end{array}$ & 40 & $\begin{array}{l}6.37 \\
( \pm 0.57)\end{array}$ & 9 \\
\hline Se- & & $\begin{array}{l}2.10 \\
( \pm 0.53)\end{array}$ & 25 & $\begin{array}{l}7.93 \\
( \pm 0.61)\end{array}$ & 8 & $\begin{array}{l}3.94 \\
( \pm 3.05)\end{array}$ & 77 & $\#$ & $\begin{array}{l}4.61 \\
( \pm 0.71)\end{array}$ & 15 & $\begin{array}{l}3.49 \\
( \pm 0.51)\end{array}$ & 15 & $\begin{array}{l}4.22 \\
( \pm 0.97)\end{array}$ & 23 \\
\hline
\end{tabular}

\# Crop was not harvested but biomass was cut and removed in June. Manually tilled so that soil was bare fallow during summer 